\title{
A Two-Mode Clustering Method to Capture the Nature of the Dominant Interaction Pattern in Large Profile Data Matrices
}

\author{
Jan Schepers \\ Katholieke Universiteit Leuven and Maastricht University
}

\author{
Iven Van Mechelen \\ Katholieke Universiteit Leuven
}

\begin{abstract}
Profile data abound in a broad range of research settings. Often it is of considerable theoretical importance to address specific structural questions with regard to the major pattern as included in such data. A key challenge in this regard pertains to identifying which type of interaction (double ordinal, mixed ordinal/disordinal, double disordinal) most adequately fits the major pattern in a profile data set at hand. In the present article a novel methodology is proposed to deal with this challenge. This methodology is based on constrained and unconstrained versions of a recently introduced 2-mode clustering model, the real-valued hierarchical classes model. The methodology is illustrated using empirical Person $\times$ Situation profile data on altruism.
\end{abstract}

Keywords: profile data, two-mode clustering, nature of interaction, constrained and unconstrained RV-HICLAS

Profile data abound in a broad range of research settings. For instance, in contextualized personality psychology (see, e.g., Mischel, 2004; Mischel \& Shoda, 1995, 1998), researchers typically study the intensity of some target behavior (e.g., level of aggression) as displayed by a set of persons in multiple situations (i.e., Person $\times$ Situation behavioral data). The goal then is to capture individual differences in "behavior-in-context," that is, in behavioral profiles across situations. Other examples of profile data include characterizations of patients on a set of clinical variables and preference profiles of customers as studied in the psychology of marketing. In these and similar examples, the data can typically be arranged into a matrix in which the rows represent individuals (patients, customers) and the columns represent variables (e.g., situations, clinical characteristics, preference objects).

A major challenge when dealing with profile data is to capture the structural information as included in them (e.g., the structure of individual differences in behavior-in-context in the case of contextualized personality psychology). However, profile data typically pertain to a large number of persons and a large number of variables (e.g., situations). Consequently, the amount of information as included in such data is simply overwhelming. When looking for structural information, one may therefore wish to focus on major patterns as included in the data rather than on minor

This article was published Online First July 11, 2011.

Jan Schepers, Department of Psychology, Katholieke Universiteit Leuven, Leuven, Belgium, and Faculty of Psychology and Neuroscience, Maastricht University, Maastricht, the Netherlands; Iven Van Mechelen, Department of Psychology, Katholieke Universiteit Leuven.

The research reported in this article was partially supported by the Research Council of Katholieke Universiteit Leuven (GOA/2005/04) and by the Fund for Scientific Research-Flanders (Project G.0146.04).

Correspondence concerning this article should be addressed to Jan Schepers, Faculty of Psychology and Neuroscience, Maastricht University, P.O. Box 616, 6200 MD Maastricht, the Netherlands. E-mail: jan.schepers@ maastrichtuniversity.nl details. For this purpose one may wish to rely on the rich family of two-mode clustering methods (for a structured overview of this family, see Van Mechelen, Bock, \& De Boeck, 2004). When applied to profile data, two-mode clustering methods yield a simultaneous clustering of the persons into person types (or person classes) and of the variables (e.g., situations) into variable classes. A simultaneous clustering may further go with a reduced profile matrix that yields values for each person type with regard to all variable classes. This reduced profile matrix may be considered a summary representation, which may unveil the major pattern in the data by removing particularities that show up for a small number of persons and/or variables (e.g., situations) only.

Beyond simply capturing the major pattern as included in profile data, it is often of considerable theoretical importance to address specific structural questions with regard to this pattern (see, e.g., Mischel, 2009; Mischel \& Shoda, 2008; Van Mechelen, 2009). To illustrate, each of the panels of Figure 1 displays behavior intensity profiles (also called behavioral signatures) of three person classes across a set of four situation classes as included in a hypothetical reduced profile matrix.

Each profile implies a rank ordering of the situation classes in terms of the intensity with which the behavior under study is elicited. An important structural question then is whether this rank ordering is consistent across all person classes. If this is the case, as in Panels A, C, and D of Figure 1, and unlike in Panel B, then the situation classes can be ordered on a single dimension; this could further be interpreted as an underlying situational force that elicits behavior. For example, in the case of aggression such a situational force could pertain to the amount of frustration as implied by each situation. Furthermore, each situation class also implies a rank ordering of the person classes in terms of the intensity with which the behavior under study is displayed. One may then consider the consistency of the person class rank orderings across situation classes as well. If consistency holds true (such as in Panel C, in which the profiles do not intersect), then this implies that the persons can be ordered on a single underlying 

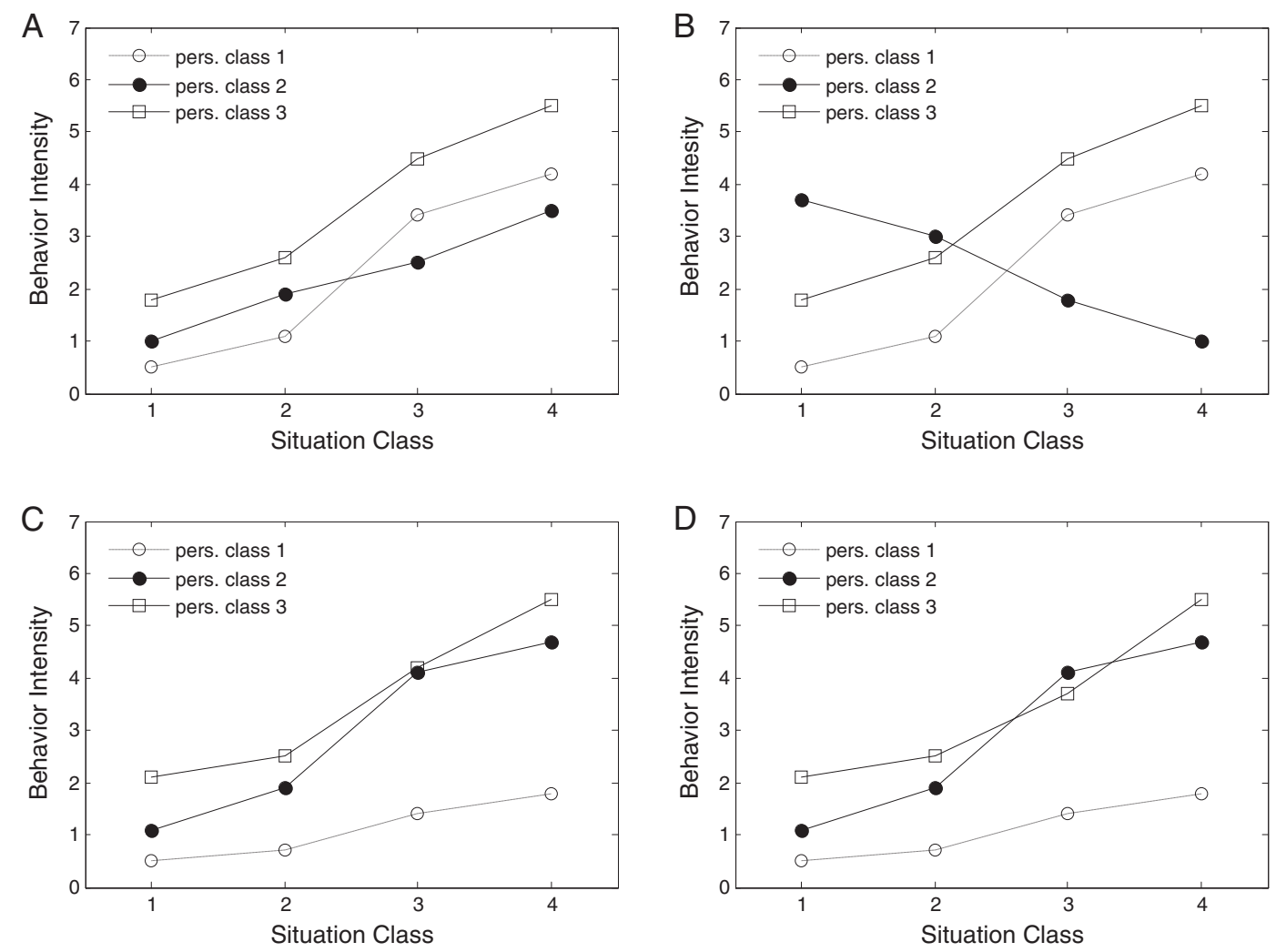

Figure 1. Behavior intensity profiles of three person classes (pers. class) across a set of four situation classes as included in a hypothetical reduced profile matrix. In terms of elicited behavioral intensity, the rank order of the situation classes is consistent across all person classes in Panels A, C, and D, whereas the rank ordering of the person classes is consistent across all situation classes in Panel B only.

person dimension, linking up with a generalized trait model account of individual differences (see, e.g., Marshall \& Brown, 2006). For example, in the case of aggression, such a person dimension may be frustration tolerance. Van Mechelen (2009) discussed more in detail a number of theoretical implications of the presence/absence of a consistent person and/or situation class rank ordering in contextualized personality psychology.

The issue of whether the person and/or variable classes display consistent rank orderings on the level of the major pattern as included in reduced profile data can be formalized in terms of the nature of the interaction between person and variable classes. In particular, if consistency of rank orderings of variable classes across person classes holds true, then with respect to the variable classes the interaction is ordinal, and disordinal otherwise (Lubin, 1961). For instance, with respect to situation classes, the interaction is ordinal in Panels A, C, and D of Figure 1 and disordinal in Panel B. Similarly, if consistency of the rank orderings of the person classes across the variable classes holds true, then with respect to the person classes the interaction is ordinal, and disordinal otherwise. For instance, with respect to the person classes, the interaction is disordinal in Panels A, B, and D and ordinal in Panel C.

Clearly, an interaction may be ordinal with respect to none, one, or both types of classes (Aiken \& West, 1991; Hager \& Westermann, 1983; Mokkan \& Lewis, 1982; Shaffer, 1991). This implies that four types of two-way interactions may be distinguished (see Table 1).

Obviously, violations of rank order consistency may occur because of small differences between only a few entries of the reduced profile matrix of a two-mode clustering of profile data. As an example, one may look at Panel D of Figure 1. The structure in this panel is almost identical to the double ordinal structure of Panel C, apart from a minor reversal of the rank order between Person Classes 2 and 3 in Situation Class 3. In this and similar cases, it may be most interesting to retrieve whether smaller reversals, which typically show up in unconstrained two-mode clustering solutions (because of the flexibility in the underlying model), are to be taken seriously or rather are to be considered as ignorable, minor departures from ordinality. Unfortunately, how-

Table 1

Four Types of Interactions in the Case of a Two-Mode Clustering of Person by Variable Profile Data

\begin{tabular}{lll}
\hline & \multicolumn{2}{c}{ Variable class } \\
\cline { 2 - 3 } Person class & \multicolumn{1}{c}{ Disordinal } & \multicolumn{1}{c}{ Ordinal } \\
\hline Disordinal & Double disordinal & Disordinal-ordinal \\
Ordinal & Ordinal-disordinal & Double ordinal \\
\hline
\end{tabular}


ever, to date no suitable methodology is available to answer this question and therefore to distinguish between the four types of interaction on the level of the major pattern in a profile data matrix.

In the present article, a novel methodology is proposed to deal with this challenge. This methodology is based on constrained and unconstrained versions of a recently introduced two-mode clustering model, the real-valued hierarchical classes (RV-HICLAS) model (Schepers \& Van Mechelen, 2010). Like other two-mode clustering methods, RV-HICLAS models of profile data imply a simultaneous reduction of the sets of persons and variables into a limited number of person and variable classes. The key novelty as proposed in the present article is the possibility of constraining the classes of either type to be totally ordered on a single underlying dimension, without requiring any a priori information on the ordering in question; rather, such information is induced from the data. Finally, by fitting a series of constrained as well as unconstrained RV-HICLAS models it is possible to identify which type of interaction (as included in Table 1) most adequately fits the major pattern in the profile data matrix at hand.

The remainder of this article is organized as follows: First, the RV-HICLAS model is recapitulated. Next, constrained versions of this model that link up with the four interaction types as included in Table 1 are introduced, and an RV-HICLAS-based strategy that can capture the nature of a two-way interaction in a profile data set at hand is outlined. Subsequently, the proposed strategy is applied to a data set on altruism. The article ends with some concluding remarks.

\section{RV-HICLAS}

\section{Model}

RV-HICLAS is rooted in the family of hierarchical classes (HICLAS) models (De Boeck \& Rosenberg, 1988). The HICLAS family comprises a group of two-mode clustering methods for Person $\times$ Variable profile data matrices D (e.g., binary Person $\times$ Item success/failure observations, rating-valued or continuous Person $\times$ Situation emotion intensity scores). Those methods are based on a decomposition of the profile data under study. This decomposition is somewhat similar to the decomposition of a profile data matrix $\mathbf{D}$ in a principal components model into a matrix of component scores $\mathbf{A}$ and a matrix of component loadings $\mathbf{B}, \mathbf{D}=\mathbf{A} \mathbf{B}^{\prime}$, but with the role of the component scores now being taken over by person cluster memberships and the role of the component loadings by variable cluster memberships (Van Mechelen, Lombardi, \& Ceulemans, 2007). Successful applications of HICLAS models can be found in a variety of research domains within psychology, including the study of psychopathology and clinical diagnosis (Gara, Silver, Escobar, Holman, \& Waitzkin, 1998; Van Mechelen \& De Boeck, 1989; Woolfolk et al., 1999), the psychology of individual differences (De Boeck \& Van Mechelen, 1990; Vansteelandt \& Van Mechelen, 2006), and self-perception and identity (Reich, Silbert-Mazzarella, Spence, \& Siegel, 2005; Reid \& Deaux, 1996; Stirratt, Meyer, Ouellette, \& Gara, 2008).

Within the HICLAS family, Schepers and Van Mechelen (2010) have recently proposed the RV-HICLAS model for positive realvalued profile data matrices (as a generalization of the HICLAS model for rating-valued profile data matrices; see Van Mechelen et al., 2007). An RV-HICLAS model for an $I \times J$ Person $\times$ Variable profile data matrix $\mathbf{D}$ with entries $d_{i j}$ implies (a) a binary $I \times P$ person cluster matrix $\mathbf{A}$, which contains the memberships $a_{i p}(1=$ yes, $0=$ no) of the $I$ persons in each of $P$ (possibly overlapping) person clusters; (b) a binary $J \times Q$ variable cluster matrix $\mathbf{B}$, which contains the memberships $b_{j q}(1=$ yes, $0=$ no) of the $J$ variables in each of $Q$ (possibly overlapping) variable clusters; and (c) a positive real-valued $P \times Q$ matrix $\mathbf{G}$ that links the person and variable clusters, with entry $g_{p q}$ representing the reconstructed value of the $q$ th variable cluster for the $p$ th person cluster. Note that the values of the ordered pair $(P, Q)$, which are to be prespecified, denote the complexity or rank of the RV-HICLAS model, similar to the number of components in a principal components model (but now with two numbers being needed, because in an RV-HICLAS model the number of person clusters may differ from the number of variable clusters). Making use of the matrices $\mathbf{A}, \mathbf{B}$, and $\mathbf{G}$, along with an $I \times J$ residual matrix $\mathbf{E}$ (with entries $e_{i j}$ ), RV-HICLAS implies the following decomposition of the profile data matrix D (Schepers \& Van Mechelen, 2010; Schepers, Van Mechelen, \& Ceulemans, in press):

$$
d_{i j}=\hat{d}_{i j}+e_{i j}=\underset{p=1}{P} \underset{q=1}{Q} \max _{q=1} a_{i p} b_{j q} g_{p q}+e_{i j},
$$

where $\hat{\mathbf{D}}$ denotes an $I \times J$ reconstructed data matrix with entries $\hat{d}_{i j}$, and max denotes the maximum operator.

Table 2 shows a hypothetical profile data matrix $\mathbf{D}$, and Table 3 the three matrices $\mathbf{A}, \mathbf{B}$, and $\mathbf{G}$ of a rank $(3,2)$ RV-HICLAS model that, according to Equation 1, reconstructs the data $\mathbf{D}$ of Table 2 exactly (i.e., $\forall i, j: e_{i j}=0$ ).

As illustrated by Table 3 , the person and variable clusters in an RV-HICLAS model are overlapping, indeed, because a single person/variable can belong to several person/variable clusters. However, from the cluster membership patterns, partitionings of the persons and the variables into person and variable classes can be identified. In particular, a person class then consists of all persons who share the same cluster membership pattern across the person clusters. A similar definition holds for the variable classes. In Table 3, for instance, one may derive from matrix $\mathbf{A}$ that there are three person classes (i.e., $\left\{\mathrm{p}_{1}, \mathrm{p}_{6}\right\},\left\{\mathrm{p}_{2}, \mathrm{p}_{3}, \mathrm{p}_{7}, \mathrm{p}_{8},\right\}$, and $\left\{\mathrm{p}_{4}\right.$, $\left.\left.p_{5}\right\}\right)$ and from matrix $\mathbf{B}$ that there are three variable classes (i.e., $\left\{\mathrm{v}_{1}, \mathrm{v}_{3}\right\},\left\{\mathrm{v}_{2}, \mathrm{v}_{5}, \mathrm{v}_{7}\right\}$, and $\left.\left\{\mathrm{v}_{4}, \mathrm{v}_{6}\right\}\right)$.

Table 2

Hypothetical Profile Data Matrix D

\begin{tabular}{cccccccc}
\hline & \multicolumn{7}{c}{ Variable } \\
\cline { 2 - 8 } Person & $\mathrm{v}_{1}$ & $\mathrm{v}_{2}$ & $\mathrm{v}_{3}$ & $\mathrm{v}_{4}$ & $\mathrm{v}_{5}$ & $\mathrm{v}_{6}$ & $\mathrm{v}_{7}$ \\
\hline $\mathrm{p}_{1}$ & 2.0 & 1.5 & 2.0 & 2.0 & 1.5 & 2.0 & 1.5 \\
$\mathrm{p}_{2}$ & 3.4 & 3.4 & 3.4 & 3.1 & 3.4 & 3.1 & 3.4 \\
$\mathrm{p}_{3}$ & 3.4 & 3.4 & 3.4 & 3.1 & 3.4 & 3.1 & 3.4 \\
$\mathrm{p}_{4}$ & 4.2 & 3.4 & 4.2 & 3.4 & 4.2 & 4.2 & 3.4 \\
$\mathrm{p}_{5}$ & 4.2 & 3.4 & 4.2 & 3.4 & 4.2 & 4.2 & 3.4 \\
$\mathrm{p}_{6}$ & 2.0 & 1.5 & 2.0 & 2.0 & 1.5 & 2.0 & 1.5 \\
$\mathrm{p}_{7}$ & 3.4 & 3.4 & 3.4 & 3.1 & 3.4 & 3.1 & 3.4 \\
$\mathrm{p}_{8}$ & 3.4 & 3.4 & 3.4 & 3.1 & 3.4 & 3.1 & 3.4 \\
\hline
\end{tabular}


Table 3

Person Cluster Matrix A, Variable Cluster Matrix B, and Linking Matrix $\boldsymbol{G}$ of Exact Rank $(3,2)$ RV-HICLAS Decomposition of the Profile Data From Table 2

\begin{tabular}{|c|c|c|c|c|c|c|c|}
\hline \multirow{2}{*}{$\begin{array}{l}\text { Person, variable, or } \\
\text { person cluster }\end{array}$} & \multicolumn{3}{|c|}{$\mathbf{A}$} & \multicolumn{2}{|c|}{ B } & \multicolumn{2}{|c|}{ G } \\
\hline & $\overline{\mathrm{PC}_{1}}$ & $\mathrm{PC}_{2}$ & $\overline{\mathrm{PC}_{3}}$ & $\overline{\mathrm{VC}_{1}}$ & $\overline{\mathrm{VC}_{2}}$ & $\overline{\mathrm{VC}_{1}}$ & $\overline{\mathrm{VC}_{2}}$ \\
\hline \multicolumn{8}{|l|}{ Person } \\
\hline $\mathrm{p}_{1}$ & 1 & 0 & 0 & & & & \\
\hline $\mathrm{p}_{2}$ & 1 & 0 & 1 & & & & \\
\hline $\mathrm{p}_{3}$ & 1 & 0 & 1 & & & & \\
\hline $\mathrm{p}_{4}$ & 1 & 1 & 1 & & & & \\
\hline $\mathrm{p}_{5}$ & 1 & 1 & 1 & & & & \\
\hline $\mathrm{p}_{6}$ & 1 & 0 & 0 & & & & \\
\hline $\mathrm{p}_{7}$ & 1 & 0 & 1 & & & & \\
\hline $\mathrm{p}_{8}$ & 1 & 0 & 1 & & & & \\
\hline \multicolumn{8}{|l|}{ Variable } \\
\hline $\mathrm{v}_{1}$ & & & & 1 & 1 & & \\
\hline$v_{2}$ & & & & 1 & 0 & & \\
\hline $\mathrm{v}_{3}$ & & & & 1 & 1 & & \\
\hline $\mathrm{v}_{4}$ & & & & 0 & 1 & & \\
\hline $\mathrm{v}_{5}$ & & & & 1 & 0 & & \\
\hline $\mathrm{v}_{6}$ & & & & 0 & 1 & & \\
\hline $\mathrm{v}_{7}$ & & & & 1 & 0 & & \\
\hline \multicolumn{8}{|l|}{ Person cluster } \\
\hline $\mathrm{PC}_{1}$ & & & & & & 1.5 & 2.0 \\
\hline $\mathrm{PC}_{2}$ & & & & & & 2.1 & 4.2 \\
\hline $\mathrm{PC}_{3}$ & & & & & & 3.4 & 3.1 \\
\hline
\end{tabular}

Furthermore, it follows from the decomposition rule seen in Equation 1 that all persons of the same person class take the same reconstructed data values on all variables. Similarly, all variables of the same variable class display the same reconstructed data values across all persons. (All this can also be easily verified in Table 2.) This further implies that an RV-HICLAS model goes with a reduced profile matrix that contains the reconstructed data values for each person class across all variable classes. To illustrate, Table 4 contains the reduced profile matrix as associated with the RV-HICLAS model of Table 3.

An important characteristic of HICLAS models that distinguishes them from other two-mode clustering methods is that they represent so-called hierarchical relations between person classes and between variable classes. A person class is hierarchically

Table 4

Reduced Profile Matrix Associated With the RV-HICLAS Model of Table 3

\begin{tabular}{lccc}
\hline & \multicolumn{3}{c}{ Variable class } \\
\cline { 2 - 4 } Person class & $\left\{\mathrm{v}_{1}, \mathrm{v}_{3}\right\}$ & $\left\{\mathrm{v}_{2}, \mathrm{v}_{5}, \mathrm{v}_{7}\right\}$ & $\left\{\mathrm{v}_{4}, \mathrm{v}_{6}\right\}$ \\
\hline$\left\{\mathrm{p}_{1}, \mathrm{p}_{6}\right\}$ & 2.0 & 1.5 & 2.0 \\
$\left\{\mathrm{p}_{2}, \mathrm{p}_{3}, \mathrm{p}_{7}, \mathrm{p}_{8}\right\}$ & 3.4 & 3.4 & 3.1 \\
$\left\{\mathrm{p}_{4}, \mathrm{p}_{5}\right\}$ & 4.2 & 3.4 & 3.4 \\
\hline
\end{tabular}

Note. $\mathrm{RV}$-HICLAS $=$ real-valued hierarchical classes; $\mathrm{p}=$ person; $\mathrm{v}=$ variable. below another one if in the reduced profile matrix all the entries of the row corresponding to the first person class are less than or equal to the corresponding entries of the row corresponding to the second person class. For instance, from Table 4 one may derive that person class $\left\{p_{1}, p_{6}\right\}$ is hierarchically below person class $\left\{p_{2}\right.$, $\left.\mathrm{p}_{3}, \mathrm{p}_{7}, \mathrm{p}_{8}\right\}$. The hierarchical relation between the variable classes is defined similarly: A variable class is hierarchically below another variable class if in the reduced profile matrix all the entries of the column corresponding to the first variable class are less than or equal to the corresponding entries of the column corresponding to the second variable class. For instance, from Table 4 one may derive that variable class $\left\{v_{2}, v_{5}, v_{7}\right\}$ is hierarchically below variable class $\left\{\mathrm{v}_{1}, \mathrm{v}_{3}\right\}$. The hierarchical relations between the person and variable classes are further represented in a straightforward way by the person and variable cluster membership matrices $\mathbf{A}$ and $\mathbf{B}$ of the RV-HICLAS model. In particular, it holds that two person classes are hierarchically related if their cluster membership patterns in $\mathbf{A}$ are in a subset-superset relation. For instance, one may verify in Table 3 that this is the case, indeed, for person classes $\left\{\mathrm{p}_{1}, \mathrm{p}_{6}\right\}$ and $\left\{\mathrm{p}_{2}, \mathrm{p}_{3}, \mathrm{p}_{7}, \mathrm{p}_{8}\right\}$. Similarly, it holds that two variable classes are hierarchically related if their cluster membership patterns in $\mathbf{B}$ are in subset-superset relation.

One may note that the hierarchical relations between classes as just discussed can be related to the four types of interaction in Table 1 (this is discussed more later). In this regard, the person and variable clusters are to be considered as tools that allow the representation of the hierarchical relations. As a consequence, the 
primary focus of our methodology is on the cluster membership patterns of the person and variable classes rather than on the person and variable clusters themselves.

\section{Estimation}

The hypothetical profile data matrix $\mathbf{D}$ of Table 2 can be reconstructed exactly by the rank $(3,2)$ RV-HICLAS model of Table 3. However, in empirical applications, where the number of rows and columns of a profile data matrix $\mathbf{D}$ are typically large, it is unreasonable to expect that one may perfectly reconstruct $\mathbf{D}$ with an RV-HICLAS solution of limited rank. Instead, nonzero residuals $e_{i j}$ are to be allowed between the observed data values $d_{i j}$ and the reconstructed data values $\hat{d}_{i j}$. An RV-HICLAS solution then is to be estimated that implies an optimal approximate reconstruction of the observed data, optimality being defined in the least squares sense. In particular, for a profile data set at hand and a to-be-prespecified rank $(P, Q)$, a least squares RV-HICLAS estimate $(\mathbf{A}, \mathbf{B}, \mathbf{G})$ minimizes the loss function

$$
f=\sum_{i=1}^{I} \sum_{j=1}^{J}\left(d_{i j}-\hat{d}_{i j}\right)^{2},
$$

where $\hat{d}_{i j}$ can be reconstructed exactly by means of $(\mathbf{A}, \mathbf{B}, \mathbf{G})$ using Equation 1.

Unlike in, for instance, least squares regression analysis, the solution that minimizes Equation 2 cannot be found by mathematical derivation but instead, as with most clustering techniques, requires resorting to an optimization algorithm. For fitting a rank $(P, Q) \mathrm{RV}$-HICLAS model to a profile data set at hand, a two-stage algorithm has been proposed that combines two well-known optimization approaches: simulated annealing (SA) and alternating least squares (ALS). This combined approach has been shown to yield good estimates (Ceulemans, Van Mechelen, \& Leenen, 2007).

The first stage of the algorithm is an SA step, and the second one an ALS step based on a deterministic local descent that starts from the solution returned by the SA step. The two-stage algorithm starts from a randomly chosen initial solution. In order to avoid ending up in a locally optimal solution, a multistart procedure is employed, which implies that the algorithm is run multiple times, each time starting from a different initial solution, with the best result from all runs being retained as the final estimate.

Any SA algorithm starts from some initial solution to the optimization problem and then "jumps' from one solution to a randomly selected new trial solution. However, in order to jump from the current solution to a trial solution, the latter must be in the neighborhood of the current solution. After every jump, it is evaluated whether the trial solution is better than the current one in terms of loss function (see Equation 2). If this is the case, the algorithm accepts the trial solution as the new current solution and subsequently makes a new jump from that new current solution. If the trial solution is not better than the current solution, the algorithm may still accept the trial solution as the new current solution, but only with a probability that is inversely related to the difference in loss between the current and trial solutions (for more details, see, e.g., Aarts \& Lenstra, 1997). If the trial solution is not accepted, another randomly selected trial solution from the neigh- borhood is considered as a candidate to replace the current solution. For a particular optimization problem at hand, one must decide how to define solution neighborhoods. A natural choice in the case of RV-HICLAS is to define a neighborhood for a given current solution as the set of all other solutions that differ from the current solution with respect to a single row of one of the cluster matrices $\mathbf{A}$ or $\mathbf{B}$ or with respect to a single entry of the linking matrix G. During the annealing process, the probability of accepting worse solutions gradually decreases such that, at the end, only better solutions are accepted to replace the current one.

In the second (ALS) stage of the algorithm, trial solutions worse than the current solution are no longer accepted. Furthermore, unlike in SA, the ALS procedure does not jump from a current solution to a trial solution that is randomly selected from within a neighborhood of the current solution but rather to the best possible trial solution in the neighborhood, provided that it implies an improvement in terms of loss function (see Equation 2). In particular, the iterative procedure starts from an initial solution $\left(\mathbf{A}^{0}, \mathbf{B}^{0}\right.$, $\mathbf{G}^{0}$; as resulting from the SA stage) and, at iteration $m$, constructs a conditional estimate $\mathbf{A}^{m}$ of the person cluster matrix (given $\mathbf{A}^{m-1}, \mathbf{B}^{m-1}$, and $\mathbf{G}^{m-1}$ ); for this purpose, it makes use of a separability property of the loss function (De Boeck \& Rosenberg, 1988). This means that the cluster matrix $\mathbf{A}^{m}$ does not need to be estimated as a whole but rather row by row, which considerably reduces the complexity of the optimization. For HICLAS models, these row-wise estimates can be obtained as the solution to a regression problem with binary constraints on the coefficients (for further details, see Ceulemans, Van Mechelen, \& Leenen, 2003; Leenen and Van Mechelen, 2001). Next, a conditional estimate $\mathbf{B}^{m}$ of the situation cluster matrix (given $\mathbf{A}^{m}, \mathbf{B}^{m-1}$, and $\mathbf{G}^{m-1}$ ) is obtained similarly. Finally, the linking matrix $\mathbf{G}^{m}$ is estimated conditionally (given $\mathbf{A}^{m}, \mathbf{B}^{m}$, and $\mathbf{G}^{m-1}$ ) by means of a state-ofthe-art multivariate optimization algorithm (sequential quadratic programming; see, e.g., Nocedal \& Wright, 1999). The ALS iterations are further repeated until no more improvement in the value of the loss function is observed.

\section{A Method to Reveal the Nature of Two-Way Interactions}

\section{Representation of Interactions by RV-HICLAS Model}

We start this section by examining how the different types of interaction as included in Table 1 are represented by the RVHICLAS model. Throughout, we keep using the model of Table 3 as a guiding example.

To find out which type of interaction is represented by an RV-HICLAS model, one has to inspect the reconstructed profile data matrix that goes with the model or, equivalently (and more parsimoniously), the corresponding reduced profile matrix. In our example, the latter matrix can be found in Table 4. On the one hand, one has to check in this reduced profile matrix whether the interaction is ordinal with respect to the person classes, that is, whether the rank order of the person classes (in terms of the associated reconstructed data values) is consistent across the variable classes. On the other hand, one has to check whether the interaction is ordinal with respect to the variable classes, that is, whether the rank order of the variable classes is consistent across the person classes. In the 
example of Table 4, it appears that, on the one hand, the rank order of the person classes is consistent across the variable classes; indeed, by denoting rank order by $\leq$ in all variable classes it holds that $\left\{\mathrm{p}_{1}, \mathrm{p}_{6}\right\} \leq\left\{\mathrm{p}_{2}, \mathrm{p}_{3}, \mathrm{p}_{7}, \mathrm{p}_{8}\right\} \leq\left\{\mathrm{p}_{4}, \mathrm{p}_{5}\right\}$. On the other hand, the rank order of the variable classes is not consistent across the person classes; as for person class $\left\{\mathrm{p}_{1}, \mathrm{p}_{6}\right\}$ it holds that $\left\{\mathrm{v}_{2}, \mathrm{v}_{5}\right.$, $\left.\mathrm{v}_{7}\right\}<\left\{\mathrm{v}_{4}, \mathrm{v}_{6}\right\}$, whereas for person class $\left\{\mathrm{p}_{2}, \mathrm{p}_{3}, \mathrm{p}_{7}, \mathrm{p}_{8}\right\}$ it holds that $\left\{v_{2}, v_{5}, v_{7}\right\}>\left\{v_{4}, v_{6}\right\}$. Hence, the interaction as included in the RV-HICLAS model of our example is ordinal-disordinal in nature.

One may now take one step further to examine how the type of interaction is being represented by the RV-HICLAS model. A key observation at this point is that consistency of the rank order of the person (respectively variable) classes is reflected immediately in the hierarchical relations as represented by the RV-HICLAS model. Indeed, the rank order of the person (respectively variable) classes is consistent if and only if the hierarchy of the classes in question constitutes a total order or a chain; such a total order is further represented by the corresponding cluster matrix in the RV-HICLAS model taking the pattern of a Guttman scale (Guttman, 1944). For example, the person cluster matrix A in Table 3 is a Guttman scale (reflecting the ordinality of the interaction with respect to the person classes); this can be easily verified after permuting the rows and columns of $\mathbf{A}$ as shown in Table 5. Yet, in the same example, the variable cluster matrix $\mathbf{B}$ is not a Guttman scale, reflecting the fact that the hierarchy is not a total order (with variable class $\left\{v_{2}, v_{5}, v_{7}\right\}$ being neither hierarchically below nor hierarchically above variable class $\left\{\mathrm{v}_{4}, \mathrm{v}_{6}\right\}$ ).

\section{Constrained RV-HICLAS}

From what was just explained, the implication may be derived that one may force an RV-HICLAS model to represent an interaction that is ordinal with respect to the person (respectively variable) classes by allowing only those solutions to be estimated in which the corresponding cluster matrix takes a Guttman scale pattern. Conversely, if no such Guttman constraint is imposed on a cluster matrix, the RV-HICLAS model is allowed to represent an interaction that is disordinal with respect to the corresponding class type. This implies that four types of (un)constrained RVHICLAS models can be formulated in which, on the one hand, the

Table 5

Person Cluster Matrix A of Table 3 in Which Rows and Columns Are Rearranged to Reflect Its Guttman Scale Pattern

\begin{tabular}{cccc}
\hline & \multicolumn{3}{c}{$\mathbf{A}$} \\
\cline { 2 - 4 } Person & $\mathrm{PC}_{1}$ & $\mathrm{PC}_{3}$ & $\mathrm{PC}_{2}$ \\
\hline $\mathrm{p}_{1}$ & 1 & 0 & 0 \\
$\mathrm{p}_{6}$ & 1 & 0 & 0 \\
$\mathrm{p}_{2}$ & 1 & 1 & 0 \\
$\mathrm{p}_{3}$ & 1 & 1 & 0 \\
$\mathrm{p}_{7}$ & 1 & 1 & 0 \\
$\mathrm{p}_{8}$ & 1 & 1 & 0 \\
$\mathrm{p}_{4}$ & 1 & 1 & 1 \\
$\mathrm{p}_{5}$ & 1 & 1 & 1 \\
\hline
\end{tabular}

Note. $\mathbf{A}=$ person cluster matrix with memberships of the persons in three person clusters; $\mathrm{PC}=$ person cluster. person cluster matrix $\mathbf{A}$ and, on the other hand, the variable cluster matrix $\mathbf{B}$ are constrained or not. These four types of models thus correspond to the four types of interaction as included in Table 1. By fitting these four (un)constrained RV-HICLAS models to a profile data set at hand and by comparing their results (see next subsection), one may unveil the dominant type of interaction as present in the data.

The estimation of constrained RV-HICLAS models that include Guttman constraints on either one or both of the cluster matrices comes down to reducing the solution space of the RV-HICLAS analysis to the subset of solutions that satisfy the constraints in question. Technically speaking, in the SA this comes down to restricting neighborhoods of solutions, whenever applicable, to solutions that preserve the Guttman constraint; in the ALS stage of the algorithm the procedure for finding conditionally optimal cluster matrices is restricted in a similar way.

One may note that, in the terminology developed by Ceulemans, Van Mechelen, and Kuppens (2004), the Guttman constraints are structure constraints, because they pertain to the structure of a particular subset of parameters of the model rather than to their actual values. Stated differently, the Guttman constraint implies that a total ordering is forced on the classes, without any prespecification of which person/variable belongs to which class or which class is to be assigned to which position in the total order; all of this information is to be inferred from the data during the dataanalytic process.

\section{RV-HICLAS-Based Strategy}

To reveal the nature of the interaction on the level of the major pattern in a profile data matrix under study, one may then proceed as follows. The general idea is that, within a range of ranks, one looks for the specific (constrained or unconstrained) RV-HICLAS model that most adequately describes the data at hand. This is a model selection problem that can be tackled by means of a two-step strategy. The first step pertains to determining the rank of the model in question; in the second step, given the rank resulting from the first step, one determines which type of (un)constrained RV-HICLAS model best captures the major pattern in the profile data matrix. The following paragraphs explain each of these steps in more detail.

The first step in this strategy concerns rank selection. This problem may be solved by first fitting, for a broad range of different possible ranks, an unconstrained RV-HICLAS model to the data (note that the unconstrained model includes all constrained ones as special cases). Subsequently, within the resulting set of fitted models, the most adequate one is selected. This is achieved on the basis of a procedure that is similar to the wellknown scree test as used in principal component analysis to decide upon the number of components. The basic idea is that the most adequate solution is one with a good fit but with not too large a rank. Because the rank of a number of HICLAS models (including RV-HICLAS) is not expressed as a single number, the scree test cannot be readily applied in this case. As a way out, we relied on a generalized scree test as proposed by Ceulemans and Van Mechelen (2005) for rank selection in the HICLAS family (a method that has also been shown to work well within the context of rank selection for other, closely related, decomposition models; Ceulemans \& Kiers, 2006; Schepers, Ceulemans, \& Van Mechelen, 2008). Specifically, for a set of solutions of different 
ranks, the fit is plotted against the total number of clusters (i.e., $P+Q$ ) involved in the solution in question, with fit being measured as the proportion of explained variation, as seen in

$$
f i t=1-\frac{\sum_{i=1}^{I} \sum_{j=1}^{J}\left(d_{i j}-\hat{d}_{i j}\right)^{2}}{\sum_{i=1}^{I} \sum_{j=1}^{J}\left(d_{i j}-d_{. .}\right)^{2}},
$$

where $d_{\text {. }}$ denotes the average value of the profile data matrix $\mathbf{D}$ (for a graphical illustration, see Figure 2). Next, solutions that lie on the higher boundary of the convex hull of this plot (which, in Figure 2, is displayed by a solid line) are retained. Finally, the solution that has an optimal ratio of gain in fit over increase in complexity is selected. For this purpose, for each solution on the boundary line, the ratio between the slopes from the previous solution to the present and from the present to the next, is calculated (for further details, see Ceulemans \& Van Mechelen, 2005). In Figure 2, the optimal ratio is found at the point where the total number of clusters equals 5 .

In the second step of the strategy, the four types of (un)constrained RV-HICLAS models corresponding to the four types of interaction as included in Table 1 are fitted to the data set at hand in the rank resulting from the first step. To further choose between the four types of fitted (un)constrained RV-HICLAS models, one might be tempted to simply select the RV-HICLAS model with the best fit. However, such a strategy would consistently lead to selecting the fully unconstrained model because this includes all other models as special cases; as such, it does not properly deal with the risk of overfitting. Cross-validation can be used to overcome this. In particular, we recommend using the $K$-fold crossvalidation method as proposed before by Louwerse, Smilde, and Kiers (1999); in the past, this method has been shown to work well for selecting from among different types of constrained dimensional reduction models that are closely related to RV-HICLAS (Timmerman \& Kiers, 2003). The $K$-fold cross-validation procedure as applied here implies that the observed profile data matrix is randomly divided into $K$ equally sized parts that constitute a partition of the set of all entries of the profile data matrix $\mathbf{D}$. The

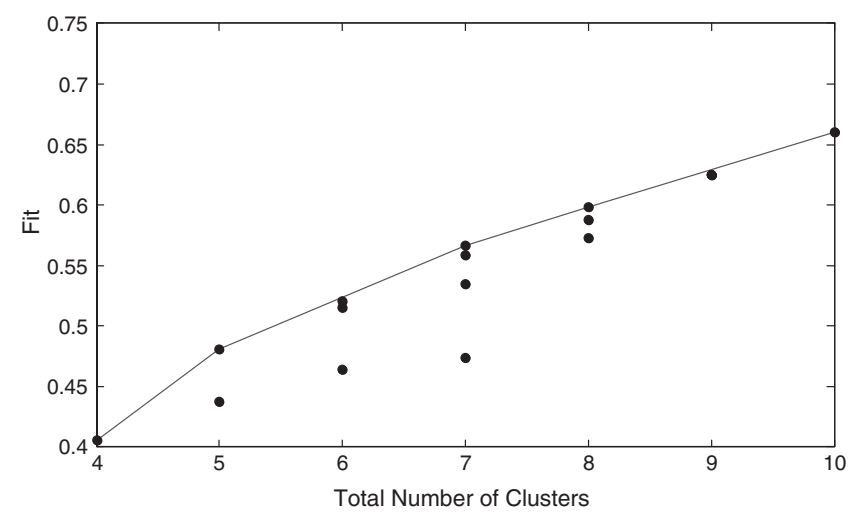

Figure 2. A generalized scree plot for a set of 16 solutions of differing rank for a data set at hand. Each point represents the fit of a single solution. idea then is to predict the data in each part (dealt with as a test or validation set) on the basis of an RV-HICLAS model that is estimated on the basis of only the other $K-1$ parts of the data (dealt with as a training set). This allows one to estimate to what degree the results of an analysis generalize to an independent (i.e., validation) data set. The prediction error of the model when predicting the $k$ th data part is computed as the sum of squared differences between the actual observed data entries in that part and the corresponding reconstructed values resulting from the estimated model (based on the other $K-1$ parts of the data), making use of the decomposition rule (see Equation 1). The procedure is repeated for all $K$ validation sets (implying that all data entries are used for both training and validation and that each data entry is used for validation exactly once). Finally, in order to obtain a global measure of the predictive validity of the model at hand, the $K$ estimates of prediction error are summed, yielding the predicted residual error sum of squares (PRESS). Of the four (un)constrained RV-HICLAS models, the one with the smallest PRESS is selected. One may note that the proposed cross-validation procedure also serves as a protection against the selection of a solution that is heavily affected by one or only a few influential observations in the data. After all, such solutions will perform relatively poor in validation sets, resulting in larger PRESS values.

\section{An Empirical Application to Altruism}

In this section, the RV-HICLAS-based strategy to capture the nature of the dominant interaction in profile data is illustrated using data from the domain of contextualized personality psychology (Mischel \& Shoda, 1995, 1998), which aims at characterizing individual differences in behavioral profiles across situations. A critical challenge in this regard is to capture Person $\times$ Situation interactions as included in behavioral profile data. The nature of such interactions may hint at the underlying mechanism through which the behavior under study is shaped (Van Mechelen, 2009).

The data of our application stem from a study on altruism (Quintiens, 1999). The key question that goes with these data is to retrieve the mechanism underlying individual differences in helping behavior. A group of 102 students was presented with a set of 16 vignettes, each of which described in a few sentences an emergency situation that typically occurs in the everyday life of students, with a victim who could possibly be helped by the participant. This is an (abbreviated) example of a situation description: "In a very crowded grocery store you see a little boy, weeping and crying for his mum." The students were asked to rate each situation with respect to the extent to which they would be willing to help the victim in it. For this purpose they had to use a rating scale ranging from 0 (definitely not) to 6 (definitely yes).

In order to capture the nature of the dominant interaction in the Student $\times$ Situation willingness to help profile matrix, the RVHICLAS-based strategy as proposed earlier was applied. First, in view of the rank selection, unconstrained RV-HICLAS models with ranks ranging from $(2,2)$ up to $(5,5)$ were estimated (each using 50 multistarts) for the $102 \times 16$ profile data matrix $\mathbf{D}$. Figure 2 is the associated generalized scree plot; the upper boundary of the convex hull of this plot is represented by a solid line. The optimal ratio of gain in fit over increase in complexity is found at the point where the total number of clusters equals five. There are two solutions with five clusters in total, namely, the solutions of 
rank $(2,3)$ and of rank $(3,2)$. The solution of rank $(2,3)$ is the one with the highest fit (i.e., 0.46) on the higher boundary of the convex hull. We therefore proceed with RV-HICLAS solutions of rank $(2,3)$, that is, solutions with 2 person and 3 situation clusters.

Next, keeping the rank fixed at $(2,3)$, all four types of (un)constrained RV-HICLAS models were fitted to the data. To decide upon which of these models was to be preferred, the $K$-fold cross-validation method as described earlier was applied (with $K=10$, as recommended by Hastie, Tibshirani, \& Friedman, 2001). The PRESS values associated to each of the (un)constrained RV-HICLAS models are displayed in Table 6. As can be seen in this table, the two models with a constrained person cluster matrix are clearly preferred over the two other models. With regard to the situation cluster matrix, the results are less compelling (i.e., compare 2,731 with 2,713). However, it also appears that the estimated situation cluster matrix of the unconstrained model takes the form of a Guttman scale, without being forced to do so. This strongly suggests that the fully constrained model (and thus a double ordinal interaction) in rank $(2,3)$ most adequately captures the major pattern in $\mathbf{D}$. Table 7 contains reduced versions of the cluster membership matrices $\mathbf{A}, \mathbf{B}$, and the linking matrix $\mathbf{G}$ of this model.

Figure 3 shows, for the two person classes, the behavioral profiles across the three situation classes, along with a $95 \%$ confidence interval around each estimated profile value (based on the data values of all person-situation combinations to which the profile value pertains). In order to derive a substantive psychological interpretation for the situation Guttman scale, the position on it (quantified as 1,2,3) was correlated with external ratings of the situations as obtained from expert judges. A high correlation was obtained with expert ratings of the extent to which the situation was emotionally threatening for the potential helper $(r=-.73)$, with situations of class $S_{1}$ (which elicit the least amount of willingness to help) being considered as emotionally more threatening. Note that this correlational analysis makes sense only because the situation classes are totally ordered. The position on the person Guttman scale further appeared to relate to dispositional ratings of perceived self-efficacy (Bandura, 1977) with respect to helping others, $F(1,100)=11.58, p<.001$, with students of class $P_{2}$ considering themselves as more efficacious in helping others. It therefore appears that individuals are more willing to help if on the one hand the situation does not put a negative emotional burden on them as a potential helper and if, on the other hand, they believe they can actually make a difference.

Beyond the dominant interaction in these data being identified as of a double ordinal type, it is interesting that the difference between the two person classes is larger in the situation class that takes an intermediate position on the situation Guttman scale than

Table 6

PRESS Values Associated to Each of the (Un)Constrained Rank (2, 3) RV-HICLAS Models as Fitted to the Altruism Data

\begin{tabular}{ccc}
\hline & \multicolumn{2}{c}{ Situation cluster matrix } \\
\cline { 2 - 3 } Person cluster matrix & Unconstrained & Constrained \\
\hline Unconstrained & 2,875 & 2,880 \\
Constrained & 2,731 & 2,713 \\
\hline
\end{tabular}

Note. $\quad$ PRESS $=$ predicted residual error sum of squares; RV-HICLAS $=$ real-valued hierarchical classes. in the other two situation classes, $t(1626)=-2.52, p<.01$. Stated differently, the difference in willingness to help between the high and low efficacious students is larger in the moderately threatening situations compared with those having a low or high level of threat. As such, this links up with the type of interaction found by Marshall and Brown (2006), which implies that individual differences in behavior are more pronounced in ambiguous situations.

\section{Discussion}

\section{Relevance of the Interaction Issue}

Many psychological research domains other than contextualized personality psychology give rise to profile data for which the diagnosis of the nature of the dominant two-way interaction (i.e., double ordinal, mixed ordinal/disordinal, double disordinal) is of key importance. Next, we illustrate with two examples.

The first example is found in the domain of psychopathology. Suppose that a group of depressed patients is scored on a set of clinical variables. An interesting question then is whether differences between the depressed patients can be captured by a single underlying quantitative dimension (i.e., severity of depression) or rather that those differences relate to qualitatively different subtypes of depression. Obviously, a total order of the patients on a single (quantitative) dimension corresponds to a rank ordering of the patients that is consistent across the clinical variables and, hence, to an interaction that is ordinal with respect to the patients. Conversely, the presence of qualitatively different subtypes of depression corresponds to an interaction that is disordinal with respect to the patients (or patient subtypes). Otherwise, our proposed method not only provides an answer to the question of whether the differences between patients are quantitative or qualitative in nature but also yields summary information on the contents of these differences (e.g., in terms of reduced clinical variable profiles for different subtypes of depression).

The second example is situated in the field of marketing. Suppose a company plans to develop a new advertisement campaign for one of its products. To determine which life aspects (e.g., luxury, freedom, health) the campaign should focus on, it may first present a group of consumers with a set of different such aspects and ask each of them to rate each aspect in terms of the amount of value they put on it. The question of whether the rank order of the life aspects is consistent across all consumers (i.e., whether the interaction is ordinal with respect to the life aspects) in the resulting Consumer $\times$ Aspect profile data is most consequential in this case. Indeed, disordinality would imply the existence of market segments that differ in terms of the rank order of the life aspects they care about. This may imply a need for different optimal advertisement strategies as a function of which market segment is targeted.

\section{Data Requirements}

The proposed method requires fully crossed Person $\times$ Variable profile data. This implies that, apart from any incidental missingness, the method is limited to cases in which an observation is available for each combination of a person and a variable. Structural missingness (e.g., when some variables have been measured for one subset of persons and other variables for a different subset 
Table 7

Reduced Person and Situation Cluster Membership Matrices $\boldsymbol{A}$ and $\boldsymbol{B}$ and Linking Matrix $\boldsymbol{G}$ of Fully Constrained Rank (2,3) RV-HICLAS Solution for the Altruism Profile Data

\begin{tabular}{|c|c|c|c|c|c|c|c|c|}
\hline \multirow{2}{*}{$\begin{array}{l}\text { Person class, situation class, or } \\
\text { person cluster }\end{array}$} & \multicolumn{2}{|c|}{$\mathbf{A}$} & \multicolumn{3}{|c|}{ B } & \multicolumn{3}{|c|}{ G } \\
\hline & $\mathrm{PC}_{1}$ & $\mathrm{PC}_{2}$ & $\mathrm{SC}_{1}$ & $\mathrm{SC}_{2}$ & $\mathrm{SC}_{3}$ & $\mathrm{SC}_{1}$ & $\mathrm{SC}_{2}$ & $\mathrm{SC}_{3}$ \\
\hline \multicolumn{9}{|l|}{ Person class } \\
\hline $\mathrm{P}_{1}(n=44)$ & 1 & 0 & & & & & & \\
\hline $\mathrm{P}_{2}(n=58)$ & 1 & 1 & & & & & & \\
\hline \multicolumn{9}{|l|}{ Situation class } \\
\hline $\mathrm{S}_{1}(n=3)$ & & & 1 & 0 & 0 & & & \\
\hline $\mathrm{S}_{2}(n=4)$ & & & 1 & 1 & 0 & & & \\
\hline $\mathrm{S}_{3}(n=9)$ & & & 1 & 1 & 1 & & & \\
\hline \multicolumn{9}{|l|}{ Person cluster } \\
\hline $\mathrm{PC}_{1}$ & & & & & & 3.09 & 4.25 & 5.78 \\
\hline $\mathrm{PC}_{2}$ & & & & & & 3.82 & 5.55 & 6.48 \\
\hline
\end{tabular}

Note. $\mathrm{RV}$-HICLAS $=$ real-valued hierarchical classes; $\mathrm{PC}=$ person cluster; $\mathrm{SC}=$ situation cluster; $\mathrm{P}=$ person class; $\mathrm{S}=$ situation class.

of persons, such as in between-subjects designs) cannot be dealt with appropriately.

Furthermore, at present, the proposed method is restricted to data matrices that pertain to two different sets of entities (say, persons and variables); technically speaking, such data may be referred to as two-way, two-mode (Carroll \& Arabie, 1980). In some research settings, however, it may be most useful to consider a third mode as well. For instance, in personality research one may be interested in the nature of the dominant interaction in Person $X$ Situation $\times$ Response profile arrays with behavioral data (i.e., in three-way, three-mode data). One may, however, note that the restriction to two-way, two-mode data is not an inherent limitation of our proposed methodology, because a three-mode extension of it could be conceived rather straightforwardly.

\section{Characteristics of the Newly Proposed Methodology}

In this subsection, we focus on our newly proposed methodology, in terms of both its intrinsic characteristics and its relation to other methods.

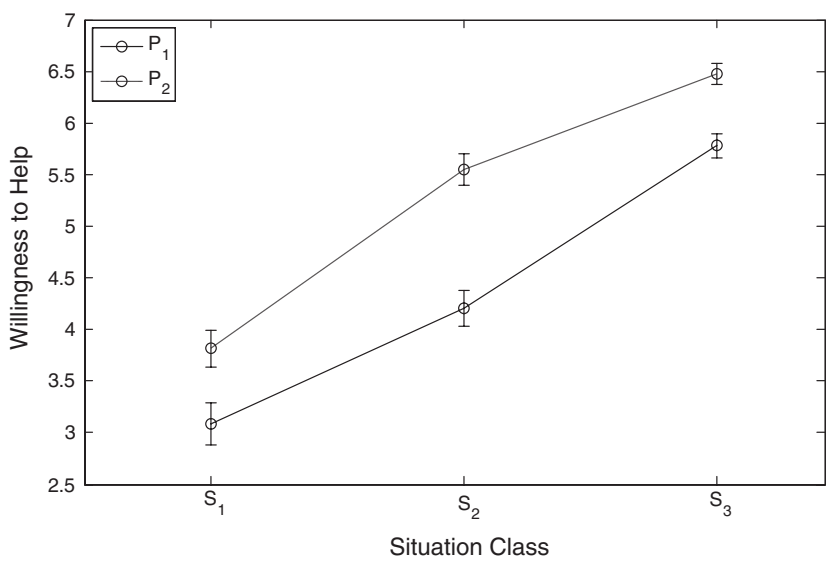

Figure 3. Degree of willingness to help as a function of person class (P) and situation class (S).
Intrinsic characteristics. The following three aspects of the methodology deserve some discussion: (1) its focus on dominant patterns, (2) its inductive nature, and (3) its deterministic character.

First, the RV-HICLAS-based strategy focuses on the dominant pattern within a data set, rather than on minor details. Within the data-analytic process, a model is sought that fits the observed data as well as possible (in a least squares sense), with in general discrepancies being allowed between the reconstructed data entries on the basis of the fitted model (i.e., $\hat{d}_{i j}$ ) and the observed data entries (i.e., $d_{i j}$ ). At this point, it is important to remark that, when RV-HICLAS models are subjected to constraints, these constraints are to be satisfied exactly on the level of the dominant pattern (i.e., on the level of the reconstructed data) but only approximately on the level of the observed data. In other words, a model can be constrained to represent an ordinal interaction with respect to one of the class types, although violations to ordinality with respect to that class type may occur in the observed data. If such violations in the observed data are to be deemed ignorable (which is decided in the model selection), one may then, however, safely conclude that the ordinality in question represents the major pattern in the data set at hand.

Second, the RV-HICLAS-based strategy includes two types of inductive aspects: It induces on the one hand which persons belong to each person class, and which variables belong to each variable class, and on the other hand also which classes are hierarchically related. No a priori information is required with regard to these two aspects. This further implies that the proposed constrained RVHICLAS models force a total order on the induced person and/or variable classes, with the classification as well as the order being induced during the data-analytic process.

Third, the RV-HICLAS-based strategy is deterministic in nature. As such, it does not involve any distributional assumptions, and the estimation of the various constrained and unconstrained models involves only minimizing a least squares loss function. To be sure, for related models it has been observed that minimizing the loss function becomes more difficult (i.e., the algorithm is more likely to end up in a locally optimal solution) in the case of simulated data that are generated on the basis of more complex stochastic mechanisms that involve, for instance, heteroscedasticity and dependencies between residuals (Schepers, Van Mechelen, \& Ceulemans, 2006). Fortu- 
nately, however, this local optimality problem can be solved by simply increasing the number of runs of the algorithm.

Relation to other methods. The following three kinds of related methods will be focused on: (1) clustering methods, (2) methods to study the nature of interactions, and (3) methods to check for order consistency.

First, RV-HICLAS is a clustering method. It is important to note that many well-known clustering methods-such as $k$ means (see, e.g., Hartigan, 1975; MacQueen, 1967), its probabilistic variant latent profile analysis (Lazarsfeld \& Henry, 1968), and hierarchical clustering (Ward, 1963) — aim at a clustering of a single set of elements only (e.g., a set of persons), whereas RV-HICLAS yields a simultaneous clustering of two sets (e.g., persons and variables). As such, RV-HICLAS belongs to the family of two-mode clustering methods (Van Mechelen et al., 2004). Within the two-mode clustering family, the distinctive and innovative characteristic of the RV-HICLAS-based strategy resides in the order constraints as imposed on the reduced profile matrix (in view of representing each of the four types of interaction in the reconstructed data).

Second, the RV-HICLAS-based methodology can be contrasted with more traditional methods used to study interactions. At this point, it may be useful to revisit the example of contextualized personality psychology. When studying Person $\times$ Situation interactions in this domain, one may opt to base the study on one or more prespecified (categorical or continuous) characteristics of the persons and situations. Making use of regression and/or analysis of variance (ANOVA) models, one may subsequently investigate how the criterion behavior of interest relates to these person and situation characteristics, and in particular also to their interaction. A limitation of such an approach, however, is that any inference about the possible Person $\times$ Situation interaction is conditional on the chosen person and situation characteristics. In this regard, it is useful to look at the Person $\times$ Situation interaction from the perspective of fully crossed Person $\times$ Situation profile data. Viewed from that perspective, limiting the study of the Person $\times$ Situation interaction to the part that can be captured by means of a few prespecified person and situation characteristics implies that one limits the study of the interaction to a small part of its degrees of freedom. In contrast, in our method the persons and situations are simply dealt with as such. This implies that we start from the full interaction (with its full number of degrees of freedom), without any a priori specification of the relevant person and situation characteristics that are involved in the Person $\times$ Situation interaction. As a matter of fact, in our methodology, the relevant characteristics are induced during the data analysis and can be derived from the output of the analysis. Otherwise, person or situation characteristics that are derived from the RV-HICLAS output could be included in subsequent regression- or ANOVA-based analyses (e.g., the classification of the persons on the one hand and of the situations on the other hand could be entered as factors in a two-factorial ANOVA). This shows that the more traditional deductive ANOVA/regression approach and our inductive strategy are not contenders but rather complement one another.

Third, our methodology can be related to other methods that focus on order relations. In particular, in the scaling domain some work has been done on examining to what degree observed data matrices satisfy a double ordinal pattern, that is, how much they differ from a perfect Guttman scale. Relevant work in this regard includes Guttman's (1950) coefficient of reproducibility and Cliff's (1977) tailored testing of order consistency, which both rely on the same concept of ordi- nality as in the present article. These methods, however, have been primarily developed for binary data; this has significant implications because in the binary case a perfect person order implies a perfect item order (and vice versa), meaning that only two types of interactions exist (viz., double disordinal and double ordinal ones). Furthermore, the methods in question focus on the Guttman scale that is closest to the full data matrix rather than on data reduction and the dominant pattern as included in the data.

\section{Software}

The RV-HICLAS-based strategy as outlined in the present article has been implemented in a MATLAB package that is freely available (along with instructions for use) from http://ppw.kuleuven.be/okp/ software/InterAct. This package does not require any specialized MATLAB skills. It can be used to identify, in a fully automated way, the nature of the interaction in the dominant pattern of a profile data matrix at hand.

\section{Summary}

Identifying the nature of the dominant interaction pattern in large profile matrices is a challenge that shows up in many domains within psychology. A novel method was presented to deal with this challenge. This method is based on constrained and unconstrained versions of a two-mode clustering method (viz., RV-HICLAS). A study of altruism, using a Person $\times$ Situation profile matrix on helping behavior, was used to demonstrate how the method can be successfully applied to empirical data. As a particular benefit, the proposed method not only yields a test of the nature of the dominant interaction pattern in the data under study but also provides an insightful reduced picture of its contents.

\section{References}

Aarts, E., \& Lenstra, J. (1997). Local search in combinatorial optimization. New York, NY: Wiley.

Aiken, L. S., \& West, S. G. (1991). Multiple regression: Testing and interpreting interactions. Newbury Park, CA: Sage.

Bandura, A. (1977). Self-efficacy: Toward a unifying theory of behavioral change. Psychological Review, 84, 191-215. doi:10.1037/0033-295X.84.2.191

Carroll, J. D., \& Arabie, P. (1980). Multidimensional scaling. Annual Review of Psychology, 31, 607-649. doi:10.1146/annurev.ps.31.020180.003135

Ceulemans, E., \& Kiers, H. A. L. (2006). Selecting among three-mode principal component models of different types and complexities: A numerical convex hull based method. British Journal of Mathematical and Statistical Psychology, 59, 133-150. doi:10.1348/000711005X64817

Ceulemans, E., \& Van Mechelen, I. (2005). Hierarchical classes models for three-way three-mode binary data: Interrelations and model selection. Psychometrika, 70, 461-480. doi:10.1007/s11336-003-1067-3

Ceulemans, E., Van Mechelen, I., \& Kuppens, P. (2004). Adapting the formal to the substantive: Constrained Tucker3-HICLAS. Journal of Classification, 21, 19-50. doi:10.1007/s00357-004-0004-1

Ceulemans, E., Van Mechelen, I., \& Leenen, I. (2003). Tucker3 hierarchical classes analysis. Psychometrika, 68, 413-433. doi:10.1007/BF02294735

Ceulemans, E., Van Mechelen, I., \& Leenen, I. (2007). The local minima problem in hierarchical classes analysis: An evaluation of a simulated annealing algorithm and various multistart procedures. Psychometrika, 72, 377-391. doi:10.1007/s11336-007-9000-9 
Cliff, N. (1977). A theory of consistency of ordering generalizable to tailored testing. Psychometrika, 42, 375-399. doi:10.1007/BF02293657

De Boeck, P., \& Rosenberg, S. (1988). Hierarchical classes: Model and data analysis. Psychometrika, 53, 361-381. doi:10.1007/BF02294218

De Boeck, P., \& Van Mechelen, I. (1990). Traits and taxonomies: A hierarchical classes approach. European Journal of Personality, 4, $147-$ 156. doi:10.1002/per. 2410040207

Gara, M. A., Silver, R. C., Escobar, J. I., Holman, A., \& Waitzkin, H. (1998). A hierarchical classes analysis (HICLAS) of primary care patients with medically unexplained somatic symptoms. Psychiatry Research, 81, 77-86. doi:10.1016/S0165-1781(98)00088-2

Guttman, L. (1944). A basis for scaling qualitative data. American Sociological Review, 9, 139-150. doi:10.2307/2086306

Guttman, L. (1950). The basis of scalogram analysis. In S. A. Stouffer (Ed.), Measurement and prediction (pp. 78-80). Princeton, NJ: Princeton University Press.

Hager, W., \& Westermann, R. (1983). Ordinality and disordinality of statistical interaction in two-way ANOVA. Archiv für Psychologie, 135, 341-359.

Hartigan, J. A. (1975). Clustering algorithms. New York, NY: Wiley.

Hastie, T., Tibshirani, R., \& Friedman, J. (2001). The elements of statistical learning. New York, NY: Springer.

Lazarsfeld, P., \& Henry, N. (1968). Latent structure analysis. Boston, MA: Houghton Mill.

Leenen, I., \& Van Mechelen, I. (2001). An evaluation of two algorithms for hierarchical classes analysis. Journal of Classification, 18, 57-80. doi: 10.1007/s00357-001-0005-2

Louwerse, D. J., Smilde, A. K., \& Kiers, H. A. L. (1999). Cross-validation of multiway component models. Journal of Chemometrics, 13, 491-510. doi:10.1002/(SICI)1099-128X(199909/10)13:5<491::AID-CEM537>3 .0. $\mathrm{CO} ; 2-2$

Lubin, A. (1961). The interpretation of significant interaction. Educational and Psychological Measurement, 21, 807-817. doi:10.1177/001316446102100406

MacQueen, J. (1967). Some methods for classification and analysis of multivariate observations. In L. M. Le Cam \& J. Neyman (Eds.), Proceedings of the Fifth Berkeley Symposium on Mathematical Statistics and Probability (pp. 281-297). Berkeley, CA: University of California Press.

Marshall, M. A., \& Brown, J. D. (2006). Trait aggressiveness and situational provocation: A test of the traits as situational sensitivities (TASS) model. Personality and Social Psychology Bulletin, 32, 1100-1113. doi: $10.1177 / 0146167206288488$

MATLAB (Version 7) [Computer software]. Natick, MA: MathWorks.

Mischel, W. (2004). Toward an integrative science of the person [Prefatory chapter]. Annual Review of Psychology, 55, 1-22. doi:10.1146/annurev .psych.55.042902.130709

Mischel, W. (2009). From Personality and Assessment (1968) to personality science, 2009. Journal of Research in Personality, 43, 282-290. doi:10.1016/j.jrp.2008.12.037

Mischel, W., \& Shoda, Y. (1995). A cognitive-affective system theory of personality: Reconceptualizing situations, dispositions, dynamics, and invariance in personality structure. Psychological Review, 102, 246268. doi:10.1037/0033-295X.102.2.246

Mischel, W., \& Shoda, Y. (1998). Reconciling processing dynamics and personality dispositions. Annual Review of Psychology, 49, 229-258. doi:10.1146/annurev.psych.49.1.229

Mischel, W., \& Shoda, Y. (2008). Toward a unified theory of personality: Integrating dispositions and processing dynamics within the cognitive-affective processing system. In O. John, R. Robins \& L. Pervin (Eds.), Handbook of Personality (3rd ed., pp. 208-241). New York, NY: Guilford Press.

Mokkan, R. J., \& Lewis, C. (1982). A nonparametric approach to the analysis of dichotomous item responses. Applied Psychological Measurement, 6, 417-430. doi:10.1177/014662168200600404

Nocedal, J., \& Wright, S. J. (1999). Numerical optimization. New York, NY: Springer. doi:10.1007/b98874
Quintiens, G. (1999). Een interactionistische benadering van individuele verschillen in helpen en laten helpen [An interactionist approach to individual differences in helping and allowing to help]. Unpublished master's thesis, Department of Psychology, Katholieke Universiteit Leuven, Leuven, Belgium.

Reich, W. A., Silbert-Mazzarella, B. A., Spence, J. A., \& Siegel, H. I (2005). Self-structure and postpartum dejection in first-time mothers. Journal of Psychology: Interdisciplinary and Applied, 139, 426-438. doi:10.3200/JRLP.139.5.426-438

Reid, A., \& Deaux, K. (1996). Relationship between social and personal identities: Segregation or integration? Journal of Personality and Social Psychology, 71, 1084-1091. doi:10.1037/0022-3514.71.6.1084

Schepers, J., Ceulemans, E., \& Van Mechelen, I. (2008). Selecting among multi-mode partitioning models of different complexities: A comparison of four model selection criteria. Journal of Classification, 25, 67-85. doi:10.1007/s00357-008-9005-9

Schepers, J., \& Van Mechelen, I. (2010). Uniqueness of real-valued hierarchical classes models. Journal of Mathematical Psychology, 54, 215 221. doi:10.1016/j.jmp.2009.12.006

Schepers, J., Van Mechelen, I., \& Ceulemans, E. (2006). Three-mode partitioning. Computational Statistics \& Data Analysis, 51, 1623-1642. doi:10.1016/j.csda.2006.06.002

Schepers, J., Van Mechelen, I., \& Ceulemans, E. (in press). The real-valued model of hierarchical classes. Journal of Classification.

Shaffer, J. P. (1991). Probability of directional errors with disordinal (qualitative) interaction. Psychometrika, 56, 29-38. doi:10.1007/BF02294583

Stirratt, M. J., Meyer, I. H., Ouellette, S. C., \& Gara, M. A. (2008). Measuring identity multiplicity and intersectionality: Hierarchical classes analysis (HICLAS) of sexual, racial, and gender identities. Self and Identity, 7, 89-111. doi:10.1080/15298860701252203

Timmerman, M. E., \& Kiers, H. A. L. (2003). Four simultaneous component models for the analysis of multivariate time series from more than one subject to model intraindividual and interindividual differences. Psychometrika, 68, 105-121. doi:10.1007/BF02296656

Van Mechelen, I. (2009). A royal road to understanding the mechanisms underlying person-in-context behavior. Journal of Research in Personality, 43, 179-186. doi:10.1016/j.jrp.2008.12.012

Van Mechelen, I., Bock, H.-H., \& De Boeck, P. (2004). Two-mode clustering methods: A structured overview. Statistical Methods in Medical Research, 13, 363-394. doi:10.1191/0962280204sm373ra

Van Mechelen, I., \& De Boeck, P. (1989). Implicit taxonomy in psychiatric diagnosis: A case study. Journal of Social and Clinical Psychology, 8, 276-287. doi:10.1521/jscp.1989.8.3.276

Van Mechelen, I., Lombardi, L., \& Ceulemans, E. (2007). Hierarchical classes modeling of rating data. Psychometrika, 72, 475-488. doi: 10.1007/s11336-007-9018-z

Vansteelandt, K., \& Van Mechelen, I. (2006). Individual differences in anger and sadness: In pursuit of active situational features and psychological processes. Journal of Personality, 74, 871-910. doi:10.1111/ j.1467-6494.2006.00395.x

Ward, J. H. (1963). Hierarchical grouping to optimize an objective criterion. Journal of the American Statistical Association, 58, 236-244. doi: $10.2307 / 2282967$

Woolfolk, R. L., Gara, M. A., Ambrose, T. K., Williams, J. E., Allen, L. A., Irvin, S. L., \& Beaver, J. D. (1999). Self-complexity and the persistence of depression. Journal of Nervous \& Mental Disease, 187, 393-399. doi:10.1097/00005053-199907000-00001

Received January 17, 2008

Revision received February 23, 2011

Accepted March 27, 2011 